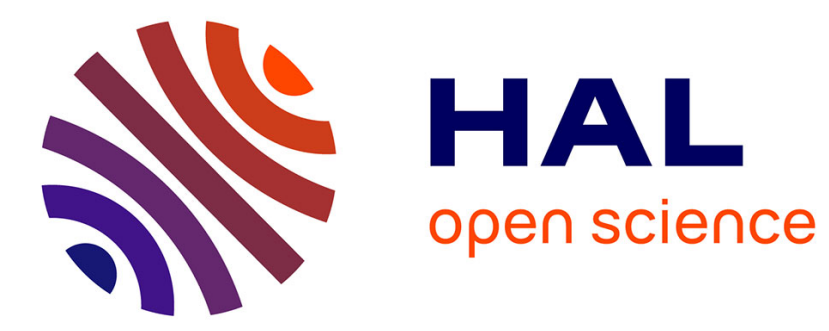

\title{
On the Advantages of Mixed-Species Groups: Impalas Adjust Their Vigilance When Associated With Larger Prey Herbivores
}

Olivier Pays, Alice Ekori, Hervé Fritz

\section{- To cite this version:}

Olivier Pays, Alice Ekori, Hervé Fritz. On the Advantages of Mixed-Species Groups: Impalas Adjust Their Vigilance When Associated With Larger Prey Herbivores. Ethology, 2014, 120 (12), pp.12071216. 10.1111/eth.12292 . hal-01502905

\section{HAL Id: hal-01502905 \\ https://hal.science/hal-01502905}

Submitted on 15 Mar 2019

HAL is a multi-disciplinary open access archive for the deposit and dissemination of scientific research documents, whether they are published or not. The documents may come from teaching and research institutions in France or abroad, or from public or private research centers.
L'archive ouverte pluridisciplinaire HAL, est destinée au dépôt et à la diffusion de documents scientifiques de niveau recherche, publiés ou non, émanant des établissements d'enseignement et de recherche français ou étrangers, des laboratoires publics ou privés. 


\title{
On the Advantages of Mixed-Species Groups: Impalas Adjust Their Vigilance When Associated With Larger Prey Herbivores
}

\author{
Olivier Pays*†, Alice Ekori†ł \& Hervé Fritz† \\ * Groupe Ecologie et Conservation, Université d'Angers, Angers, France \\ $\uparrow$ CNRS HERD programme - Hwange LTER, Hwange Main Camp Research, Hwange NP, Dete, Zimbabwe \\ \$ Laboratoire de Biométrie et Biologie Evolutive, CNRS UMR 5558, Université Claude Bernard Lyon 1, Lyon, France
}

\author{
Correspondence \\ Olivier Pays, Groupe Ecologie et \\ Conservation, Université d'Angers, Campus \\ Belle Beille, 2 Bd Lavoisier, 49045 Angers, \\ France. \\ E-mail: olivier.pays@univ-angers.fr
}

Keywords: antipredator behaviour, group living, group size, herbivores, mixed-species group, predator detection, vigilance

\begin{abstract}
Prey can obtain valuable benefits from associating with other species if heterospecifics help to detect predators or locate good food patches. In mixed-species groups, how species respond to the presence of other species remains a poorly explored question although it might give crucial insights into mechanisms underlying the interspecific coexistence. We studied temporary mixed-species groups of large herbivores in Hwange National Park (Zimbabwe) between the common impala (Aepyceros melam$p u s)$, the focal species here, and bigger species including the plains zebra (Equus quagga), the greater kudu (Tragelaphus strepsiceros) or the blue wildebeest (Connochaetes taurinus). In the Hwange savanna, the focal and smaller species are exposed to a larger range of predators than the associated species. In this context, we investigated how impalas adjusted their vigilance with group size comparing impala-only and mixed-species groups and whether the identity of heterospecifics affected vigilance of impalas. Our study showed that the time impalas spent in vigilance significantly decreased with group size when they formed impala-only groups, whereas it did not significantly vary with group size in mixed-species groups. Moreover, in mixed-species groups, impalas did not adjust their time spent in vigilance with the proportion of conspecifics and the identity of the associated species. Thus, the mechanism underlying the difference of impalas' behavioural adjustment of vigilance with group size between single- and mixed-species groups seemed to be related to the presence but not to the number and the identity of heteropecifics. Finally, we discuss the concept that larger and dominant heterospecifics were likely to increase competition for food access, thereby forcing higher vigilance of impalas, outweighing any reduction from collective vigilance.
\end{abstract}

\section{Introduction}

While animals can acquire information about the environment from their own perception, they can also acquire information from other conspecifics (Dall et al. 2005; see Goodale et al. 2010 for a review). In this context, animals can also obtain valuable benefits by being associated with other species (forming temporary or stable mixed-species groups) if the presence and behaviours of heterospecifics provide information facilitating the detection of predators (Rainey et al. 2004; Templeton \& Greene 2007; Aplin et al. 2012) or the location of potentially good foraging patches (Richter \& Tisch 1999; Stout \& Goulson 2001; but see the concept of public information in Danchin et al. 2004 and Valone 2007). Indeed, the focal and associated species may be sensitive to different cues, and the sum of information processed in a mixed-species group could make individuals more aware of their environment than they would be in single-species group (Gautier-Hion et al. 1983; Goodale \& Kotagama 2008). Moreover, it can be profitable if the focal and associated species are of the same trophic level, as the two associated species need to avoid the same 
predators and find similar resources. Such mixedspecies groups might be even more profitable when the associated species shares with the focal species several common predators but forages upon different resources. In this context, individuals of the focal species can benefit in predator detection while suffering little competition for food access (Morse 1970).

Stable mixed-species groups that persist regardless of the heterogeneity of the distribution of threats and resources are formed by many taxa including birds (Jullien \& Thiollay 1998; Sridhar et al. 2009), fish (Ward et al. 2002), insects (Menzel et al. 2008), mammals (Stensland et al. 2003) or even involving several taxa (Rasa 1983). Here, in contrast to stable groups, this article examines mixed-species groups involving two species temporarily associated while foraging. While several studies have concentrated on the antipredator advantages of mixed-species grouping in birds, ungulate associations have received relatively little attention although ungulates are often found in mixed-species groups (Washburn $\&$ De Vore 1961; Altmann \& Altmann 1970; Elder \& Eider 1970; Leuthold 1977; Gosling 1980; Sinclair 1985; FitzGibbon 1990). Indeed, in large herbivores, how the focal species responds to the presence of the associated species remains a poorly explored question although it might give crucial information on the existence of such mixed-species groups and even more on mechanisms underlying the interspecific coexistence.

Several reasons have been proposed in the literature to explain mixed-species groups (Sridhar et al. 2009; Harrison \& Whitehouse 2011; Farine et al. 2012). Studies have reported that many mammals and birds have learned to associate heterospecific alarm calls with the presence of a predator (Hauser 1988; Shriner 1998; Fichtel 2004; Rainey et al. 2004; Magrath et al. 2007). Moreover, species that normally form small groups can increase group size by heterospecific association and hence enhance the effectiveness of antipredator behaviour (as the dilution effect or collective detection) (Goodale et al. 2010). An additional benefit of mixed-species grouping may arise if predators show a preference for a particular prey species. Sinclair (1985), for example, suggested that plains zebra, Equus quagga, in the Serengeti can reduce their risk of predation by staying close to Serengeti white-bearded wildebeest, Connochaetes mearnsi, the preferred prey of large carnivores in the area. FitzGibbon (1990) reported that Grant's gazelles Nanger granti benefited from the association with Thomson's gazelles Eudorcas thomsonii because the cheetahs Acinonyx jubatus preference for the smaller Thomson's gazelles reduced the rate at which the Grant's gazelles were attacked when they formed mixed-species groups. However, while antipredator benefits might bring crucial support to our understanding of the existence of such mixed-species groups, several disadvantages can also arise. Indeed, if the associated species are socially or morphologically dominant and can increase their foraging success by kleptoparasitism of focal species or by local enhancement, heterospecific association may lead to a higher resource competition, both in terms of resource exploitation and interference competition. Accordingly, Krause \& Ruxton (2002) suggested that there were two main reasons why focal species would tolerate associated species: (1) all species would compensate by being able to reduce antipredator vigilance when associated with others; or (2) the cost of avoiding these species might be too great compared with the loss of foraging opportunities.

We studied temporary mixed-species groups of two large herbivores in Hwange National Park, Zimbabwe. In our study area, the impala (Aepyceros melampus), the focal species in this study, is temporarily associated while foraging with different associated species including the plains zebra, the greater kudu (Tragelaphus strepsiceros) or the blue wildebeest (Connochaetes taurinus). Temporary mixed-species groups are particularly interested to study from the impalas' perspective (i.e. that of the focal species) as the focal species were associated with larger body-size herbivores. Adult (male/female) impala, zebra, greater kudu and wildebeest weigh on average 60/45, 250/220, 257/ 170 and $200 / 163 \mathrm{~kg}$, respectively (Estes 1991). In the Hwange context, all of these medium-size herbivores are preyed upon by a large range of predators (Fritz et al. 2011) but impalas, the focal and smallest species, were expected to suffer from a larger range of predators than the associated species (Sinclair et al. 2003). Here, the aim of this study was to investigate whether impalas adjusted their vigilance when they formed mixed-species groups (i.e. when they were associated with one of the other species cited above). We thus compared the relationship between vigilance and group size in impalas when they formed impala-only and mixed-species groups. We also tested whether impalas adjusted their vigilance with the identity of associated species and the number of heterospecifics they were associated with.

Our predictions were the following. We hypothesized that impalas in impala-only groups should decrease their vigilance with group size if the main function of vigilance in this prey species was ascribed to predator detection (Pays et al. 2012). As the risk of predation to individuals may be reduced in large 
groups as a result of dilution (Bednekoff $\&$ Lima 1998), an individual could benefit from an increase in group size by reducing its own rate of antipredator vigilance, thus increasing its time available for feeding, without reducing the group's probability of detecting potential danger (Ale \& Brown 2007; Pays et al. 2007b). If heterospecifics were perceived as 'supplementary impalas', the mixed-species group would lead to an increase of the perceived group size (i.e. the overall group size). Thus, we would expect a negative trend between group size and vigilance, indicating a dilution-effect process (Hamilton 1971). If heterospecifics relaxed impalas in their level of perception of predation risk, we would expect under this assumption that individuals in mixed-species groups were on average less vigilant than in impala-only groups. The third assumption would consider that, while heterospecifics would relax impalas allowing them to reduce their antipredator vigilance, the associated species, morphologically dominant, would affect impalas in their food access. Indeed, the arrival of larger grazers might force impalas to leave from their current patch, if these patches are situated on the larger grazers' path. Thus, to avoid this passive interference (i.e. without aggression), impalas would increase their social monitoring in mixed-species groups. Thus, under this third assumption, we would observe two compensating mechanisms. The proportion of time impalas spent monitoring other conspecifics (to maintain social cohesion) and heterospecifics (to limit interference) would increase, whereas the proportion of time they spent scanning the environment (i.e. antipredator vigilance) would decrease with group size; as a result, overall vigilance level of impalas might not change with group size in mixed-species groups (Favreau et al. 2010). Finally, a similar pattern would be observed in mixed-species groups if impalas increased their vigilance in large groups of heterospecifics as a consequence of larger heterospecifics in mixed-species groups increasing predation risk, which may occur as lions prefer to catch larger-bodied animals (Loveridge et al. 2007a). Thus, impalas would experience an increase in the risk of being opportunistically preyed upon in the confusion of the chase if lions attack larger mixed-species groups including zebras, wildebeests or kudus.

\section{Methods}

\section{Study Area and Animals}

The fieldwork was conducted in the Main Camp area of Hwange National Park (HNP) in South-Western
Zimbabwe $\left(19^{\circ} 00^{\prime} \mathrm{S}, 26^{\circ} 30^{\prime} \mathrm{E}\right)$ from mid-Feb. to midApr. 2010. The study site is an open grassland area of ca. 64 ha surrounded by Acacia/Combretum bush, one of the plant communities characteristic of the mixedbushed grassland of the eastern Kalahari sands region (Rogers 1993). Long-term mean annual rainfall is around $606 \mathrm{~mm}$, which mainly occurs during a rainy season from the end of Oct. to the end of Apr. (Chamaillé-Jammes et al. 2007).

Observations were performed near Main Camp. In this area, the main predators of impalas comprised spotted hyenas, Crocuta crocuta, wild dogs, Lycaon pictus, leopards, Panthera pardus, cheetahs and occasionally lions, Panthera leo (Bourgarel 2004). During the wet seasons, densities of impalas, zebras, kudus and wildebeests were estimated to be $0.98 \pm 0.36$, $1.08 \pm 0.53,0.60 \pm 0.22$ and $0.23 \pm 0.11 \mathrm{ind} / \mathrm{km}^{2}$, respectively (Chamaillé-Jammes et al. 2009).

We observed 50-100 female impalas daily at the study site, 3-20 zebras, 2-12 kudus and 6-17 wildebeests mainly foraging (mostly grazing, licking saltrich soil or browsing for kudus) in the open. All female impalas in the study area formed a single clan that was divided into a variable number of groups with marked fusion-fission dynamics; this was known because about 30 adult females were ear-tagged. On the grassland, impalas occasionally form mixedspecies groups when foraging, being temporarily associated with zebras or kudus or wildebeests.

\section{Ethics Statement}

Our study was conducted under permits from the Director General of the Zimbabwe Parks and approved by the Wildlife Management Authority (Ref: D/M/ Gen/(T), Permit: 23(l) (c) (ii) 01/2010), and it complied with the current laws of Zimbabwe. The study was based only on focal sampling, and our disturbance to wildlife was consequently very limited. We did not capture and ear-tag impalas for the purpose of this study. The presence of ear-tagged impalas on the area is explained by a long-term individual-based study on population dynamics in HNP started in 2007 supervised by Dr. H. Fritz under permits from the same authorities cited above (Ref: D/M/Gen/(T): Permit: 23 (l) (c) (ii) 12/2007; 23(l) (c) (ii) 31/2007; 23(l) (c) (ii) $04 / 2009 ; 23$ (l) (c) (ii) $01 / 2010$ ).

\section{Behavioural Data}

Behavioural data were collected by focal sampling from vehicles, respecting a minimal distance of $100 \mathrm{~m}$ between the focal group and the observer to minimize 
disturbance. Animals were chosen at random for a 5-min focal sample, during the daytime. We recorded only groups whose predominant activity was foraging and which did not move far during the recording, ensuring that group size and composition did not change during the 5-min focal sample. Spatial groups were identified on the basis of a maximal separation between adjacent members of $50 \mathrm{~m}$, and on the maintenance of social and spatial cohesion of the group members during all focal sampling. There were no ambiguities in defining a group using these criteria as interindividual distances were very small. Group size was therefore taken as being equal to the size of the spatial group. We distinguished impala-only from mixed-species groups in which impalas were associated with at least one heterospecific (zebras, kudus or wildebeests). For each observation, we determined group size, the numbers of impalas and heterospecifics and the species impalas were associated with during the focal sample. The mixed-species groups that we sampled included impalas and only one of the species cited above (i.e. we sampled no mixed-species groups with three or more species).

Observations were performed in two periods only, the $3 \mathrm{~h}$ after sunrise and the $3 \mathrm{~h}$ before sundown, for two main reasons: (1) these are the daylight periods within which the main predators are active (Hunter $\delta$ Skinner 1998), and we therefore expected that impalas and other studied prey would then be likely to exhibit antipredator strategies; and (2) impalas are mainly engaged in foraging activity in these periods (Jarman \& Jarman 1973). Although we tended to limit the number of observations during any single morning or afternoon session, several individuals were sampled during some sessions. In such cases, the observer took care to avoid studying the same individual twice per session by preferentially observing tagged animals. However, although re-sampling was possible on untagged animals, we strongly believe that it represented a negligible part of our data set.

Distance to cover ( $\mathrm{D} \leq 50,50<\mathrm{D} \leq 100, \mathrm{D}>100 \mathrm{~m}$ ) was estimated while sampling individuals. It is commonly used as a proxy for predation risk (Lima 1990; Burger et al. 2000; Blumstein et al. 2003), but its role (i.e. obstructive or protective) is ambiguous for prey, and depends on many factors concerning both the predator and the prey. Expectations for the effect of distance to cover on individual vigilance are therefore not clear. However, impalas and other larger studied prey in the area including zebras and wildebeests graze in open areas, and as their main predators spend the daytime in cover and hunt from cover (e.g.
Loarie et al. 2013 for lions), we can assume that they should perceive closeness to cover as risky.

Visibility around foraging individuals provides direct information on their visual obstruction (Whittingham et al. 2004). Pays et al. (2012) showed that impalas adjusted their vigilance in relation to grass height as visibility around them varied. Therefore, we estimated the grass height of the patch in which the focal individual was foraging. We considered grass to be short when it was below the hooves of the focal impala, medium, below the upper part of the metacarpals, and tall when grass height reached the tibia, which is above their eyes when feeding. In tall grass, visibility is strongly reduced when feeding in the herb layer. Observations were not conducted when wind speeds were high.

We studied adult females of impalas to avoid any sex or age effect (Pays \& Jarman 2008). From each observation, we determined the time during which an impala was vigilant. We defined vigilance in an animal as being when it raised its head above the horizontal with marked orientation of ears, scanning its surroundings, without moving its feet. No ambiguities were encountered in distinguishing a vigilant from a non-vigilant animal. The position of the individuals within a group (i.e. peripheral or central; e.g. Blanchard et al. 2008) was impossible to determine during the sampling because the animals moved frequently when foraging. For all focal individuals, we determined the distance between the focal individual and the nearest impala (DNearImpala) $(D \leq 5, \mathrm{D}>5 \mathrm{~m})$ and, in mixed-species groups, the distance to the nearest heterospecific $(D \leq 10, \mathrm{D}>10 \mathrm{~m})$. Such interindividual classes allowed us to define unambiguous situations when animals were foraging during 5-min focal samples.

We sampled the behaviours of 56 impalas, 25 in impala-only groups and 31 in mixed-species groups comprising 11, 7 and 13 groups with zebras, wildebeests and kudus, respectively. The ranges of group sizes of impala-only and mixed-species groups were similar (i.e. from 10 to 76 and 10 to 73 individuals, respectively). In mixed-species groups, the proportion of impalas ranged from 0.16 to 0.93 .

\section{Data Analyses}

As the major aim of this study was to investigate whether impalas adjusted their vigilance with group size differently when associated with other larger prey, we used a linear fixed-effects model approach and conducted a model selection starting with a model including group size, group type (impala-only 
vs. mixed-species groups) and the interaction between these two variables (Table 1). To achieve normality and homoscedasticity and to reach linearity between our variables in all statistical procedures described above, we arcsine square root-transformed the proportion of time spent in vigilance and logtransformed the group size. To test for significance of effects, we used a backward selection procedure, beginning with the full model including the two-way interaction $\log ($ group size) $\times$ group type (model 1 , Table 1) and comparing it to the model without the interaction (model 2, Table 1) using a F test. The same procedure was applied to test for the effect of group size (model 2 vs. model 4, Table 1) and group type (model 2 vs. model 3, Table 1). We also verified that the selected model had the lowest Akaike's corrected criterion (AICc) and a $\triangle \mathrm{AICc}>2$ compared with the closest competing ones, including the null model in the comparison.

Using the same procedure (i.e. model comparison using F-value and AICc difference), we tested whether distance to the nearest impala (D $\leq 5$ vs. $\mathrm{D}>5 \mathrm{~m})$, distance to cover $(\mathrm{D} \leq 50,50<\mathrm{D} \leq 100 \mathrm{vs}$. $D>100 \mathrm{~m}$ ) and grass height (short, medium vs. tall) affected the proportion of time an impala spent in vigilance. Here, model 1 including Log(group size), group type and the two-way interaction (Table 1) was compared with the same model including one of each of the factors cited above (model 5, 6, 7, Table 1).

Finally, we investigated whether impalas in mixedspecies groups adjusted their vigilance to the proportion of impalas, the species they were associated with, and distance to the nearest heterospecific. Using the same procedure, we compared the null model (without including the Log(group size) as it did not significantly affect vigilance in mixed-species groups; see Table 3) and a model including one of the tested variable cited above (Table 3 ).
All analyses were performed in R 2.15.1 (R Development Core Team 2012).

\section{Results}

Model comparison indicated that group size and group type significantly affected the time an impala spent in vigilance (model 2, Table 1). According to the coefficients derived for each factor and the interaction group size $\times$ group type (Table 2 ), the time impalas spent in vigilance significantly decreased with group size when they formed impala-only groups but did not significantly vary with group size in mixedspecies groups, with a lower level of vigilance in mixed-groups (Fig. 1). However, Table 1 showed that neither distance to the nearest impala nor grass height significantly affected the time an impala spent in vigilance. Moreover, considering the two outcomes for model comparison between models 1 and 6 $(\mathrm{p}=0.077$ and $\triangle \mathrm{AICc}=1.73$, Table 1$)$, we did not consider that distance to cover had a significant effect on the time an impala spent in vigilance.

Finally, in mixed-species groups, impalas did not change their time spent in vigilance with the proportion of conspecifics, the species they were associated with and the distance to the nearest heterospecific (Table 3).

\section{Discussion}

Our main result indicated that the time impalas spent in vigilance significantly decreased with group size when impalas formed single-species groups, whereas it did not significantly vary with group size in mixedspecies groups. This negative trend in single-species groups has been reported in many species including birds and mammals (Pays et al. 2007a; Beauchamp 2008). As the risk of predation to individuals was

Table 1: Model comparison allowing to test for the effect of group size, group type, distance to the nearest impala, distance to cover and grass height on the arcsine square root-transformed proportion of time an impala spent in vigilance

\begin{tabular}{|c|c|c|c|c|c|c|c|c|}
\hline ID Model & Model structure & LogLike & $\mathrm{AICC}$ & $d f$ & Model comparison & $\mathrm{F}$ & df & $\mathrm{p}$ \\
\hline 0 & $\theta_{0}$ & 37.79 & -71.58 & 2 & & & & \\
\hline 1 & $\theta_{0}+\theta_{1} \cdot \log \mathrm{GS}+\theta_{2} \cdot \mathrm{GT}+\theta_{3} \cdot \mathrm{GS} \times \mathrm{GT}$ & 44.86 & -79.72 & 5 & & & & \\
\hline 2 & $\theta_{0}+\theta_{1} \cdot \operatorname{LogGS}+\theta_{2} \cdot \mathrm{GT}$ & 42.02 & -76.03 & 4 & $1-2$ & 5.556 & 1 & 0.022 \\
\hline 3 & $\theta_{0}+\theta_{1} \cdot \log G S$ & 41.07 & -76.15 & 3 & $2-3$ & 1.811 & 1 & 0.184 \\
\hline 4 & $\theta_{0}+\theta_{2} \cdot \log G T$ & 38.24 & -70.47 & 3 & $2-4$ & 7.656 & 1 & 0.008 \\
\hline 5 & $\theta_{0}+\theta_{1} \cdot \log \mathrm{GS}+\theta_{2} \cdot \mathrm{GT}+\theta_{3} \cdot \mathrm{GS} \times \mathrm{GT}+\theta_{4} \cdot$ DNearlmpala & 44.90 & -77.81 & 6 & $1-5$ & 0.085 & 1 & 0.772 \\
\hline 6 & $\theta_{0}+\theta_{1} \cdot \operatorname{LogGS}+\theta_{2} \cdot G T+\theta_{3} \cdot G S \times G T+\theta_{5} \cdot$ DCover & 47.73 & -81.45 & 7 & $1-6$ & 2.693 & 2 & 0.077 \\
\hline 7 & $\theta_{0}+\theta_{1} \cdot \log \mathrm{SS}+\theta_{2} \cdot \mathrm{GT}+\theta_{3} \cdot \mathrm{GS} \times \mathrm{GT}+\theta_{6} \cdot$ DGrassHeight & 45.12 & -76.23 & 7 & $1-7$ & 0.232 & 2 & 0.794 \\
\hline
\end{tabular}

Group size (GS) was log-transformed. Group type (GT) (impala group, mixed-species group), Distance to the nearest impala (DNearlmpala) ( $\leq \leq 5$, $\mathrm{D}>5 \mathrm{~m}$ ), distance to cover (DCover) ( $\mathrm{D} \leq 50,50<\mathrm{D} \leq 100, \mathrm{D}>100 \mathrm{~m}$ ) and grass height (GrassHeight) (short, medium and tall) were considered as categorical. Levels used as the references in the models are italicized in the legends. 
Table 2: Coefficients ( \pm SE) of the factors influencing the arcsine square root-transformed proportion of time an impala spent in vigilance

\begin{tabular}{|c|c|c|c|c|}
\hline Variables & $\begin{array}{l}\text { ID coefficient } \\
\text { of model } 1 \\
\text { in Table } 1\end{array}$ & Coefficient & SE & \\
\hline Intercept & $\theta_{0}$ & 0.731 & 0.133 & \\
\hline LogGS & $\theta_{1}$ & $-0.339 *$ & 0.091 & \\
\hline GT & $\theta_{2}$ & $-0.405^{\mathrm{ns}}$ & 0.192 & $\begin{array}{l}\text { When impala-only } \\
\text { group is the reference }\end{array}$ \\
\hline $\begin{array}{l}\text { LogGS } \\
\quad \times \text { GT }\end{array}$ & $\theta_{3}$ & $0.307 * *$ & 0.130 & $\begin{array}{l}\text { When impala-only } \\
\text { group is the reference }\end{array}$ \\
\hline
\end{tabular}

Group size (GS) was log-transformed. Group type (GT) (impala-only group, mixed-species group) was considered as categorical. Level used as the reference in the models is italicized in the legend. *** and ns indicate $0.05 \leq p<0.01,0.01 \leq p<0.001$ and non-significant, respectively (see Table 1 for statistical details).

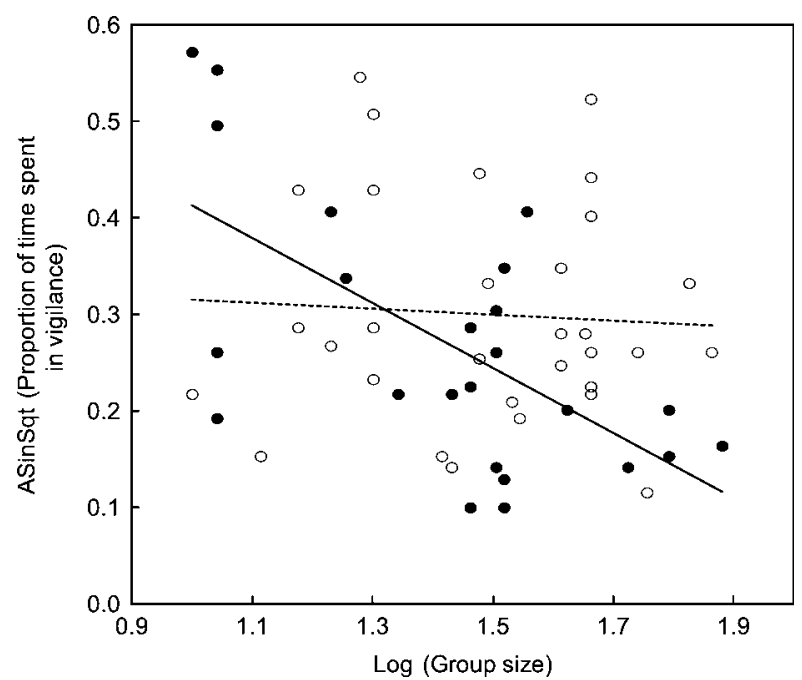

Fig. 1: Relationship between the arcsine square root-transformed proportion of time an impala spent in vigilance and the log-transformed group size in impala groups (black dots, full line) and mixed-species groups (open symbols, dashed line). Fitted curves are derived from the model 1 in the Table 1 and coefficients in Table 2. reduced in large groups as a result of dilution (Bednekoff \& Lima 1998), impalas tended to reduce their own rate of antipredator vigilance when group size increased, thus increasing their time available for feeding, without reducing the group's probability of detecting potential danger (Ale \& Brown 2007; Pays et al. 2007b). Although vigilance might also serve other functions, including detection of potential competitors (Slotow \& Rothstein 1995; Cresswell 1997), assessment of patch quality (Templeton $\delta$ Giraldeau 1996; Smith et al. 1999), searching for mates (Burger $\&$ Gochfeld 1988), and location of food patches discovered by companions (Coolen et al. 2001), this negative trend of vigilance with group size indicated that vigilance in prey species of such body size was mainly used for antipredator detection (Pulliam 1973). This result was expected as (1) populations of African ungulates like impala, in communities rich in predators, are usually top-down regulated, rather than bottom-up (Sinclair et al. 2003), (2) predator off-take on mesoherbivores in the Hwange system were abundant (Fritz et al. 2011), and (3) impalas were preyed upon by a large range of predators including spotted hyaenas, lions, leopards, cheetahs, African wild dogs as well as black-backed jackals, Canis mesomelas, for young impalas (Bourgarel 2004; Pays et al. 2012).

The time impalas spent in vigilance did not significantly vary with group size in mixed-species groups. This result does not the support the assumption that heterospecifics were simply perceived as 'supplementary impalas'. As we observed that impalas spent more time in vigilance in small- to medium-impala-only groups than in small- to medium-mixed-species groups (Fig. 1), our results support the assumption that heterospecifics relaxed impalas in their level of perception of predation risk. However, the result of the interaction between group size and group type suggests that this pattern is valid only up to 21 individuals (i.e. when $\log$ (group size) $=1.32$ in Fig. 1) and apparently reversed beyond this threshold group

Table 3: Model comparison allowing to test for the effect of group size, proportion of impalas, the species with which impalas were associated and distance to the nearest heterospecific on the arcsine square root-transformed proportion of time an impala spent in vigilance in mixed-species groups

\begin{tabular}{|c|c|c|c|c|c|c|c|c|}
\hline ID Model & Model structure & LogLik & $\mathrm{AlCC}$ & df & Model comparison & $\mathrm{F}$ & $\mathrm{df}$ & $p$ \\
\hline 8 & $\theta_{0}$ & 23.34 & -42.68 & 2 & & & & \\
\hline 9 & $\theta_{0}+\theta_{1} \cdot \log G S$ & 23.40 & -40.79 & 3 & $8-9$ & 0.114 & 1 & 0.738 \\
\hline 10 & $\theta_{0}+\theta_{2} \cdot$ Proplmpala & 23.44 & -38.88 & 4 & $8-10$ & 0.071 & 1 & 0.793 \\
\hline 11 & $\theta_{0}+\theta_{3} \cdot$ Species & 25.92 & -39.84 & 5 & $8-11$ & 1.532 & 2 & 0.230 \\
\hline 12 & $\theta_{0}+\theta_{2} \cdot$ DNearHeteroSp & 23.51 & -39.01 & 4 & $8-12$ & 0.195 & 1 & 0.664 \\
\hline
\end{tabular}

Group size of mixed-species groups (GS) was log-transformed. Proplmpala was the proportion of impalas in mixed-species groups. Mixed-species groups (Species) (Impalas associated with zebras, wildebeests or kudus) and distance to the nearest heterospecific (DNearHeteroSp) (D $\leq 10$, D $>10 \mathrm{~m}$ ) were considered as categorical. Levels used as the references in the models are italicized in the legends. 
size. While other species would relax impalas in their investment in vigilance in small- to medium-size groups, they would tend to make impalas maintain a higher vigilance level in large mixed-species groups. This result supported previous theoretical models (Beauchamp 2001) and empirical observations in single-species groups of mammals (Treves 1999). For instance, it has been reported that although eastern grey kangaroos, Macropus giganteus, spent on average most of their vigilance time in antipredator vigilance (about $75 \%$ of the overall vigilance), their proportion of time spent looking at other group members increased with group size, while the proportion of time they spent scanning the environment decreased with group size; as a result, overall vigilance levels did not change with group size (Favreau et al. 2010). Our result here showed that such a mechanism would apply for the focal species in mixed-species groups. In future studies, while impalas are less vigilant in large groups of conspecifics, it would be interesting to sample more small mixed-species groups (even rare in nature) to confirm that impalas would be less vigilant in this context. Moreover, it would be interesting to investigate the target of each vigilance act exhibited by individuals [following Favreau et al.'s method (2010)] to support our suggestion that the lack of a group-size effect on overall vigilance in multispecies groups is caused by an increase of time allocation to social vigilance with group size.

In mixed-species groups, impalas did not adjust their time spent in vigilance with the proportion of conspecifics. Thus, the mechanism underlying the difference of behavioural adjustment of vigilance with group size between single- and mixed-species groups in impalas seemed to be related to the presence but not to the number of heteropecifics. Forming mixedspecies groups for impalas might be profitable as it would allow impalas to reduce their level of risk endured as (1) the presence and behaviour of heterospecifics might improve predator detection (Templeton $\&$ Greene 2007) particularly if the impala population is regulated by predation (Sinclair et al. 2003), (2) collective detection with heterospecifics was expected to be higher than the one observed in an impala-only group (of a similar group size) particularly if the associated species is sensitive to different cues (Goodale \& Kotagama 2008), and (3) previous studies on the Hwange system have reported that zebras, wildebeests and kudus were the preferred prey of lions (Loveridge et al. 2007a,b). Sinclair (1985) suggested that zebra in the Serengeti tended to stay close to wildebeest to reduce their own risk of predation as wildebeest were the preferred prey of all the large car- nivore species (Kruuk 1972; Schaller 1972), and he concluded that predation pressure should cause the mixed-species herding. However, associating with a preferred prey species of lion, impalas may be exposed to more lion attacks and therefore increased the risk of being opportunistically preyed upon in the confusion of the chase. FitzGibbon (1990) reported that Thomson's gazelles in mixed-species groups shared vigilance to some extent with Grant's gazelles, leaving more time available for feeding. However, compared with joining conspecifics, FitzGibbon (1990) suggested that joining Grant's gazelles to form mixedspecies groups had few additional advantages and such associations were likely to be beneficial only when the number of conspecifics in the resulting mixed-species groups was relatively high. Thus, when they have the opportunity (i.e. when other species are in the surroundings), impalas might form multispecies groups to limit the effect of predation; more work is however needed to understand the proximate mechanisms promoting mixed-species grouping.

The lack of group-size effect on vigilance in multispecies groups, possibly caused by an increase of social monitoring with group size in multispecies groups, might explain why not all impalas form mixed-species groups with larger body-size herbivores when foraging despite an increased advantage in detecting predators. Although we did not detect any effect of species and distance to the nearest heterospecific in mixedspecies groups, impalas may suffer from being close to a heterospecific because of disturbance and competition while foraging. Impalas, because of their lower daily intake, can tolerate shorter swards and more dispersed bites than zebras, wildebeest and kudus because of body-mass allometry (Wilmshurst et al. 1999; Owen-Smith 2002). Nevertheless, both zebras and wildebeests can graze swards as short as or even shorter than impalas can (Arsenault \& Owen-Smith 2008) and hence would be strong competitors for small patches of short green flush. However, we might not expect impalas to suffer from severe competition for forage from these much larger heterospecifics if (1) impalas with their much smaller body size have much lower absolute food intake demands thereby being able to meet maintenance requirements more easily than larger-bodied herbivores on short grass (Illius \& Gordon 1987), (2) impala have more selective mouths than the other species thereby enabling them to more efficiently access quality forage that the larger species cannot, and (3) the larger species may facilitate grazing for impala by removing stems and litter thereby increasing access to basal leaves. Thus more investigations are needed here to examine the 
likelihood of the hypothesis of food competition as in our context, kudus and impalas should be dietarily more separate (than impalas and zebras or wildebeests), so the risk of foraging interference would have been less in impala-kudu groups. Further, if the mechanism underlying mixed-species grouping were not shaped by predation pressure but by resource distribution with species searching for the same food in a heterogeneously food-distributed landscape, impalas might pay a foraging cost due to interference in the temporary association with morphologically dominant associated species. It might be particularly true when food is rare in the environment or rapidly depleting within feeding patches. Consequently, even if increasing social monitoring with group size might be profitable for resource detection, impalas may be more constrained in their resource access in mixedspecies groups. However, impalas might tolerate the presence of other species as they would compensate by being able to reduce antipredator vigilance and/or the cost of avoiding associates might be too great compared with the cost of lost foraging opportunities in the patch or of movement to another patch (Krause $\&$ Ruxton 2002). In conclusion, more studies are needed to investigate (1) whether temporary mixed-species grouping allows impalas to maximize their food intake and ultimately their survival, and (2) both interference costs and predation risks advantages from mixed-species groups.

\section{Acknowledgements}

The Director General of the Zimbabwe Parks and Wildlife Management Authority is acknowledged for providing the opportunity to carry out this research. We are particularly grateful to Dr. H. Madzikanda for his long-term support and friendship, Mr. A. Musakwa, the Area Manager, for his support and understanding and to the Parks Senior ecologist G. Mtare. This study was carried out in the framework of the HERD program (Hwange Environmental Research Development), funded by the CNRS INEE ('Institut Ecologie et Environnement'), 'Zones Ateliers' programme, the Agence National de la Recherche FEAR project (ANR-08-BLAN-0022), the CIRAD and the French 'Ministère des Affaires Etrangères', and the 'Ambassade de France au Zimbabwe'. We would also like to thank the whole HERD team including Arnold Winston Tshipa for his crucial help in collecting data, Peter Jarman and Damien Farine for helpful comments on an earlier version of the manuscript and John Fryxell and one anonymous referee for their helpful comments.

\section{Literature Cited}

Ale, S. B. \& Brown, J. S. 2007: The contingencies of group size and vigilance. Evol. Ecol. Res. 9, 1263-1276.

Altmann, S. A. \& Altmann, J. 1970: Baboon Ecology. Univ. of Chicago Press, Chicago.

Aplin, L. M., Farine, D. R., Morand-Ferron, J. \& Scheldon, B. C. 2012: Social networks predict patch discovery in a wild population of songbirds. Proc. Roy. Soc. B 279, 4199-4205.

Arsenault, R. \& Owen-Smith, N. 2008: Resource partitioning by grass height among grazing ungulates does not follow body size relation. Oikos 117, 1711-1717.

Beauchamp, G. 2001: Should vigilance always decrease with group size? Behav. Ecol. Sociobiol. 51, 473-582.

Beauchamp, G. 2008: What is the magnitude of the groupsize effect on vigilance? Behav. Ecol. 19, 1361-1368.

Bednekoff, P. A. \& Lima, S. L. 1998: Randomness, chaos and confusion in the study of antipredator vigilance. Trends Ecol. Evol. 13, 284-287.

Blanchard, P., Sabatier, R. \& Fritz, H. 2008: Within-group spatial position and vigilance: a role also for competition? The case of impalas with a controlled food supply. Behav. Ecol. Sociobiol. 62, 1863-1868.

Blumstein, D. T., Daniel, J. C. \& Sims, R. A. 2003: Group size but not distance to cover influences agile wallaby (Macropus agilis) time allocation. J. Mammal. 84, 197204.

Bourgarel, M. 2004: Approche de la dynamique des populations de grands herbivores dans une aire protégée: L'exemple de l'impala (Aepyceros melampus) au Zimbabwe. Doctoral thesis, Université Claude Bernard, Lyon I.

Burger, J. \& Gochfeld, M. 1988: Effects of group size and sex on vigilance in ostriches (Struthio camelus): antipredator strategy or mate competition? Ostrich $\mathbf{5 9}$, $14-20$.

Burger, J., Safina, C. \& Gochfeld, M. 2000: Factors affecting vigilance in springbok: importance of vegetative cover, location in herd, and herd size. Acta Ethol. 2, 97 -104 .

Chamaillé-Jammes, S., Fritz, H. \& Murindagomo, F. 2007: Detecting climate changes of concerns in highly variable environment: quantiles regressions reveal that droughts worsen in Hwange National Park, Zimbabwe. J. Arid Environ. 71, 321-326.

Chamaillé-Jammes, S., Valeix, M., Bourgarel, M., Murindagomo, F. \& Fritz, H. 2009: Seasonal density estimates of common large herbivores in Hwange National Park, Zimbabwe. Afr. J. Ecol. 47, 804-808.

Coolen, I., Giraldeau, L.-A. \& Lavoie, M. 2001: Head position as an indicator of producer and scrounger tactics in a ground feeding bird. Anim. Behav. 61, 895-903.

Cresswell, W. 1997: Interference competition at low competitor densities in blackbirds Turdus merula. J. Anim. Ecol. 66, 461-471. 
Dall, S. R. X., Giraldeau, L.-A., Olsson, O., McNamara, J. M. \& Stephens, D. W. 2005: Information and its use by animals in evolutionary ecology. Trends Ecol. Evol. 20, $187-193$.

Danchin, E., Giraldeau, L. A., Valone, T. J. \& Wagner, R. H. 2004: Public information: from nosy neighbors to cultural evolution. Science 305, 487-491.

Elder, W. H. \& Eider, N. L. 1970: Social groupings and primate associations of the bushbuck (Tragelaphus scriptus). Mammalia 34, 356-362.

Estes, R. D. 1991: The Behavior Guide to African Mammals. Univ. of California Press, Los Angeles, CA.

Farine, D. R., Garroway, C. J. \& Sheldon, B. C. 2012: Social network analysis of mixed-species flocks: exploring the structure and evolution of interspecific social behaviour. Anim. Behav. 84, 1271-1277.

Favreau, F. R., Goldizen, A. W. \& Pays, O. 2010: Interactions among social monitoring, anti-predator vigilance and group size in eastern grey kangaroos. Proc. Roy. Soc. B 277, 2089-2095.

Fichtel, C. 2004: Reciprocal recognition of sifaka (Propithecus verreauxi verreauxi) and red-fronted lemur (Eulemur fulvus rufus) alarm calls. Anim. Cogn. 7, 45-52.

FitzGibbon, C. D. 1990: Mixed-species grouping in Thomson's and Grant's gazelles: the antipredator benefits. Anim. Behav. 39, 1116-1126.

Fritz, H., Loreau, M., Chamaillé-Jammes, S., Valeix, M. \& Clobert, J. 2011: A food web perspective on large herbivore community limitation. Ecography 34, $196-202$.

Gautier-Hion, A., Quris, R. \& Gautier, J. P. 1983: Monospecific vs polyspecific life: a comparative study of foraging and antipredatory tactics in a community of Cercopithecus monkeys. Behav. Ecol. Sociobiol. 12, 325 -335 .

Goodale, E. \& Kotagama, S. W. 2008: Response to conspecific and heterospecific alarm calls in mixed-species bird flocks of a Sri Lankan rainforest. Behav. Ecol. 19, 887894.

Goodale, E., Beauchamp, G., Magrath, R. D., Nieh, J. C. \& Ruxton, G. D. 2010: Interspecific information transfer influences animal community structure. Trends Ecol. Evol. 25, 354-361.

Gosling, L. M. 1980: Defense guilds of savannah ungulates as a context for scent communication. Symp. Zool. Soc. Lond. 45, 195-212.

Hamilton, W. D. 1971: Geometry for the selfish herd. J. Theor. Biol. 31, 295-311.

Harrison, N. M. \& Whitehouse, M. J. 2011: Mixed-species flocks: an example of niche construction? Anim. Behav. 81, 675-682.

Hauser, M. D. 1988: How infant vervet monkeys learn to recognize starling alarm calls - the role of experience. Behaviour 105, 187-201.
Hunter, L. T. B. \& Skinner, J. D. 1998: Vigilance behavior in african ungulates: the role of predation pressure. Behaviour 135, 195-211.

Illius, A. W. \& Gordon, I. J. 1987: The allometry of food intake in grazing ruminants. J. Anim. Ecol. 56, 989999.

Jarman, M. V. \& Jarman, P. J. 1973: Daily activity of impala. Afr. J. Ecol. 11, 75-92.

Jullien, M. \& Thiollay, J. M. 1998: Multi-species territoriality and dynamics in neotropical forest understorey bird flocks. J. Anim. Ecol. 67, 227-252.

Krause, J. \& Ruxton, G. D. 2002: Living in Groups. Oxford Univ. Press, Oxford.

Kruuk, H. 1972: The Spotted Hyena. Univ. of Chicago Press, Chicago.

Leuthold, W. 1977: African Ungulates: a Comparative Review of Their Ethology and Behavioral Ecology. Springer-Verlag, New York, NY.

Lima, S. L. 1990: Protective cover and the use of space: different strategies in finches. Oikos 58, 151-158.

Loarie, S. R., Tambling, C. J. \& Asner, G. P. 2013: Lion hunting behaviour and vegetation structure in an African savanna. Anim. Behav. 85, 899-906.

Loveridge, A. J., Davidson, Z., Hunt, J. E., Valeix, M., Elliot, N. \& Stapelkamp, B. 2007a: Hwange Lion Project Annual Report 2007. Zimbabwe Parks and Wildlife Management Authority, Harare, Zimbabwe.

Loveridge, A. J., Searle, A. W., Murindagomo, F. \& Macdonald, D. W. 2007b: The impact of sport-hunting on the lion population in a protected area. Biol. Conserv. 134, 548-558.

Magrath, R. D., Pitcher, B. J. \& Gardner, J. L. 2007: A mutual understanding? Interspecific responses by birds to each other's aerial alarm calls. Behav. Ecol. 18, 944951.

Menzel, F., Linsenmair, K. E. \& Blüthgen, N. 2008: Selective interspecific tolerance in tropical CrematogasterCamponotus associations. Anim. Behav. 75, 837-846.

Morse, D. H. 1970: Ecological aspects of some mixed-species foraging flocks of birds. Ecol. Monogr. 40, $119-168$.

Owen-Smith, N. 2002: Adaptive Herbivore Ecology. From Resources to Populations in Variable Environments. Cambridge Studies in Ecology. Cambridge Univ. Press, Cambridge.

Pays, O. \& Jarman, P. J. 2008: Does sex affect individual and collective vigilance in the eastern grey kangaroo? Behav. Ecol. Sociobiol. 62, 757-767.

Pays, O., Jarman, P. J., Loisel, P. \& Gerard, J.-F. 2007a: Coordination, independence or synchronisation tendency of individual vigilance in the eastern grey kangaroo? Anim. Behav. 73, 595-604.

Pays, O., Renaud, P.-C., Loisel, P., Petit, M., Gerard, J.-F. \& Jarman, P. J. 2007b: Prey synchronise their vigilant 
behaviour with other group members. Proc. Roy. Soc. B 274, 1287-1291.

Pays, O., Blanchard, P., Valeix, M., Chamaillé-Jammes, S., Duncan, P., Périquet, S., Lombard, M., Ncube, G., Tarakini, T., Makuwe, E. \& Fritz, H. 2012: Detecting predators and locating competitors while foraging: an experimental study of a medium-sized herbivore in an African savanna. Oecologia 169, 419-430.

Pulliam, H. R. 1973: On the advantages of flocking. J. Theor. Biol. 38, 419-422.

R Development Core Team 2012: R: A Language and Environment for Statistical Computing. R Foundation for Statistical Computing. R Development Core Team, Vienna.

Rainey, H. J., Zuberbühler, K. \& Slater, P. J. B. 2004: Hornbills can distinguish between primate alarm calls. Proc. Roy. Soc. B 271, 755-759.

Rasa, O. A. E. 1983: Dwarf mongoose and hornbill mutualism in the Taru Desert, Kenya. Behav. Ecol. Sociobiol. 12, 181-190.

Richter, M. R. \& Tisch, V. L. 1999: Resource choice of social wasps: influence of presence, size and species of resident wasps. Insectes Soc. 46, 131-136.

Rogers, C. M. L. 1993: A Woody Vegetation Survey of Hwange National Park. Department of National Parks and Wildlife Management, Harare.

Schaller, G. B. 1972: The Serengeti Lion. Univ. of Chicago Press, Chicago.

Shriner, W. M. 1998: Yellow-bellied marmot and goldenmantled ground squirrel responses to heterospecific alarm calls. Anim. Behav. 55, 529-536.

Sinclair, A. R. E. 1985: Does interspecific competition or predation shape the African ungulate community? J. Anim. Ecol. 54, 899-918.

Sinclair, A. R. E., Mduma, S. \& Brashares, J. S. 2003: Patterns of predation in a diverse predator-prey system. Nature 425, 288-290.

Slotow, R. \& Rothstein, S. I. 1995: Influence of social status, distance from cover, and group size on feeding and vigilance in White-crowned Sparrows. Auk 112, 1024 1031.

Smith, J. W., Benkman, C. W. \& Coffey, K. 1999: The use and misuse of public information by foraging red crossbills. Behav. Ecol. 10, 54-62.

Sridhar, H., Beauchamp, G. \& Shanker, K. 2009: Why do birds participate in mixed-species foraging flocks? A large-scale synthesis. Anim. Behav. 78, 337-347.

Stensland, E., Angerbjörn, A. \& Berggren, P. 2003: Mixed species groups in mammals. Mammal Rev. 33, 205223.

Stout, J. C. \& Goulson, D. 2001: The use of conspecific and interspecific scent marks by foraging bumblebees and honeybees. Anim. Behav. 62, 183-189.

Templeton, J. J. \& Giraldeau, L.-A. 1996: Vicarious sampling: the use of personal and public information by starlings foraging in a simple patchy environment. Behav. Ecol. Sociobiol. 38, 105-113.

Templeton, C. N. \& Greene, E. 2007: Nuthatches eavesdrop on variations in heterospecific chickadee mobbing alarm calls. Proc. Natl Acad. Sci. USA 104, 5479—5482.

Treves, A. 1999: Within-group vigilance in red colobus and redtail monkeys. Am. J. Primatol. 48, 113-126.

Valone, T. J. 2007: From eavesdropping on performance to copying the behavior of others: a review of public information use. Behav. Ecol. Sociobiol. 62, 1-14.

Ward, A. J. W., Axford, S. \& Krause, J. 2002: Mixed-species shoaling in fish: the sensory mechanisms and costs of shoal choice. Behav. Ecol. Sociobiol. 52, 182-187.

Washburn, S. L. \& De Vore, I. 1961: The social life of baboons. Sci. Am. 204, 62-71.

Whittingham, M. J., Butler, S. J., Quinn, J. L. \& Cresswell, W. 2004: The effect of limited visibility on vigilance behaviour and speed of predator detection: implications for the conservation of granivorous passerines. Oikos 106, 377-385.

Wilmshurst, J. F., Fryxell, J. M. \& Colucci, P. E. 1999: What constrains daily intake in Thomson's gazelles? Ecology 80, 2338-2347. 